\title{
Altered cerebral regulation in type 2 diabetic patients with cardiac autonomic neuropathy
}

\author{
H. Marthol • C. M. Brown • U. Zikeli • D. Ziegler • \\ N. Dimitrov $•$ R. Baltadzhieva $\cdot$ M. J. Hilz
}

Received: 27 February 2006 / Accepted: 24 May 2006 / Published online: 29 August 2006

(C) Springer-Verlag 2006

\begin{abstract}
Aims/hypothesis Assessment of cerebral regulation in diabetic patients is often problematic because of the presence of cardiac autonomic neuropathy. We evaluated the technique of oscillatory neck suction at $0.1 \mathrm{~Hz}$ to quantify cerebral regulation in diabetic patients and healthy control subjects.

Subjects and methods In nine type 2 diabetic patients with cardiac autonomic neuropathy and 11 age-matched controls, we measured blood pressure and cerebral blood flow velocity responses to application of $0.1 \mathrm{~Hz}$ neck suction. We determined spectral powers and calculated the transfer function gain and phase shift between $0.1 \mathrm{~Hz}$ blood pressure and cerebral blood flow velocity oscillations as parameters of cerebral regulation.

Results In the patients and control subjects, neck suction did not significantly influence mean values of the RR interval, blood pressure and cerebral blood flow velocity. The powers of $0.1 \mathrm{~Hz}$ blood pressure and cerebral blood flow velocity oscillations increased in the control subjects,
\end{abstract}

H. Marthol $\cdot$ U. Zikeli $\cdot$ N. Dimitrov $\cdot$ R. Baltadzhieva $\cdot$

M. J. Hilz ( $\bowtie)$

Department of Neurology, University of Erlangen-Nuremberg,

Schwabachanlage 6,

91054 Erlangen, Germany

e-mail: max.hilz@neuro.imed.uni-erlangen.de

\section{M. Brown}

Department of Medicine, Division of Physiology,

University of Fribourg,

Fribourg, Switzerland

D. Ziegler

German Diabetes Clinic, German Diabetes Center,

Leibniz Center at the Heinrich Heine University,

Düsseldorf, Germany but remained stable in the patients. Transfer function gain remained stable in both groups. Phase shift decreased in the patients, but remained stable in control subjects.

Conclusions/interpretation The absence of an increase in the power of $0.1 \mathrm{~Hz}$ blood pressure and cerebral blood flow velocity oscillations confirmed autonomic neuropathy in the diabetic patients. Gain analysis did not show altered cerebral regulation. The decrease in phase shift in the patients indicates a more passive transmission of neck suction-induced blood pressure fluctuations onto the cerebrovascular circulation, i.e. altered cerebral regulation, in the patients, and is therefore suited to identifying subtle impairment of cerebral regulation in these patients.

Keywords Autonomic neuropathy · Heart rate variability . Phase shift · Spectral analysis · Sympathetic nervous system . Transfer function gain

\section{Abbreviations \\ CBFV Cerebral blood flow velocity}

\section{Introduction}

Patients with diabetes mellitus are at high risk of cerebrovascular complications. In patients with type 2 diabetes the incidence of stroke is two- to five-fold higher than in healthy individuals [1]. Strokes in diabetic patients are probably due to microangiopathy rather than to atherosclerosis of the large arteries or cardioembolism [2]. Moreover, it has been suggested that autonomic neuropathy is an independent risk factor for strokes in patients with type 2 diabetes [3]. Both vascular disease and autonomic dysfunction in diabetic patients might contribute to compromised 
cerebral regulation, thereby further increasing the risk of cerebrovascular complications [4]. Assessment of cerebral regulation in diabetic patients is therefore important to help identify whether there is an increased risk of cerebrovascular complications [4].

Cardiovascular autonomic neuropathy is a common complication of diabetes mellitus. Patients with type 2 diabetes have impaired heart rate responses to standard autonomic tests, a phenomenon that is associated with increased cardiovascular risk and mortality $[5,6]$. One method of assessing cardiovascular autonomic function is to stimulate the carotid baroreceptors directly using oscillatory neck suction [7]. In normal subjects, oscillations in baroreceptor activity induced by neck suction at $0.1 \mathrm{~Hz}$ induce oscillations in heart rate and blood pressure at the same frequency. We previously demonstrated that the reflex heart rate and blood pressure responses to $0.1 \mathrm{~Hz}$ oscillatory neck suction were reduced in patients with type 2 diabetes [8]. Therefore, in addition to impaired cardiovagal control, patients with type 2 diabetes also show deterioration in the regulation of peripheral vascular tone.

The term 'cerebral regulation' refers to the mechanisms by which cerebral blood flow remains relatively constant even during large variations in blood pressure. The testing of cerebral regulation is based on the evaluation of cerebrovascular responses to changes in blood pressure. So far, blood pressure changes have been induced either by drugs such as angiotensin, phenylephrine and sodium nitroprusside, or by challenge manoeuvres such as orthostatic challenge, the leg-cuff-method according to Aaslid, metronomic breathing or the Valsalva manoeuvre [9]. In patients with prominent autonomic neuropathy, compromised adaptation of heart rate and blood pressure and particularly pronounced orthostatic hypotension may provide inadequate conditions for testing of cerebral regulation. Therefore, standard manoeuvres might be unsuitable for quantifying cerebral regulation in such patients. The method of sinusoidal neck suction, however, provides a refined approach to the testing of cerebral regulation [10]. Using this approach, a neck chamber is fitted over the participants' neck and subatmospheric pressure is applied in a sinusoidal manner [11]. The subsequent rhythmical deformation of the arterial vessels alternately activates and deactivates the carotid baroreceptors. Neck suction at $0.1 \mathrm{~Hz}$ generates vasomotor fluctuations at $0.1 \mathrm{~Hz}$ not only in the peripheral but also in the cerebral blood vessels [10] and is therefore suited to evaluating the transfer of sympathetically mediated $0.1 \mathrm{~Hz}$ oscillations in blood pressure onto $0.1 \mathrm{~Hz}$ oscillations in $\mathrm{CBFV}$, i.e. cerebral regulation.

Based on the findings of impaired autonomic and vascular regulation in patients with type 2 diabetes, one might also expect cerebral regulation to be affected. In this study we therefore extended our previous observations in the 14 patients with type 2 diabetes in whom we had assessed cardiovagal and vasomotor responses to sinusoidal neck suction [8]. The main objective of the present analysis was to evaluate blood pressure and cerebral blood flow velocity signals to determine whether the behaviour of the cerebral blood vessels differs in patients with type 2 diabetes patients and definite autonomic neuropathy compared with a group of healthy control subjects.

\section{Subjects and methods}

\section{Subjects}

This study comprised an evaluation of data previously obtained from 14 patients who had been diagnosed with type 2 diabetes mellitus according to the internationally accepted criteria of the American Diabetes Association and the World Health Organization. All patients underwent a battery of standard cardiovascular reflex tests. These comprised assessment of heart rate variability at rest and during metronomic breathing, the Valsalva manoeuvre and active standing [12]. Nine of the patients fulfilled the criteria of severe cardiac autonomic neuropathy, defined as two or more pathological results from standard cardiovascular reflex testing, and were therefore included in the present analysis. The mean $\pm \mathrm{SD}$ age of the nine patients was $56.2 \pm 5.4$ years, duration of diabetes $84.1 \pm 51.3$ months, weight $81.0 \pm 17.5 \mathrm{~kg}$, height $170.8 \pm 8.5 \mathrm{~cm}$ and BMI $27.6 \pm$ $4.0 \mathrm{~kg} / \mathrm{m}^{2}$. To largely control for a possible influence of prevailing poor glycaemic control, patients with $\mathrm{HbA}_{1 \mathrm{c}}$ levels above $7.7 \%$ were excluded. The individual $\mathrm{HbA}_{1 \mathrm{c}}$ levels were $6.9 \%(n=2), 7.2 \%(n=2), 7.3 \%(n=1), 7.4 \%$ $(n=1), 7.5 \%(n=2)$ and $7.7 \%(n=1)$, respectively. Four patients were treated with insulin and five patients were on oral antidiabetic drugs such as sulfonylureas or metformin. All participants had a light meal 3 to $4 \mathrm{~h}$ before testing. To ensure the absence of hypoglycaemia within $24 \mathrm{~h}$ prior to testing, all patients self-monitored their blood glucose at least three times $1 \mathrm{~h}$ after meals. The postprandial blood glucose levels were below $7.8 \mathrm{mmol} / \mathrm{l}$, without evidence of hypoglycaemia. None of the diabetic patients showed any symptoms or signs of diabetic somatic neuropathy. Two patients occasionally suffered from symptoms of orthostatic intolerance, such as postural dizziness or blurred vision.

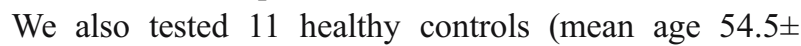
10.0 years, weight $77.7 \pm 17.2 \mathrm{~kg}$, height $170.0 \pm 7.5 \mathrm{~cm}$, BMI $26.6 \pm 4.3 \mathrm{~kg} / \mathrm{m}^{2}$ ). The controls were recruited from healthy friends and relatives of staff members and patients.

None of the participants had a history of chronic alcohol abuse, carcinoma of any origin, myopathy, hyper- or hypothyroidism, arterial hypertension, cardiac arrhythmia, 
atherosclerosis, previous kidney or pancreas transplantation, significant hepatic or renal disease or any other condition that would interfere with their ability to participate in the study. Apart from insulin or oral antidiabetics in the diabetic patients, none of the participants was on any other medication known to influence the cardiovascular or autonomic systems. The participants were instructed to refrain from nicotine, caffeine or alcohol for $24 \mathrm{~h}$ prior to the measurements.

Before the testing procedure, each control and patient underwent duplex sonographic examination of the extracranial arteries to rule out atherosclerosis and significant stenoses of the carotid arteries.

The study was approved by the University of ErlangenNuremberg Ethics Committee and written informed consent was obtained according to the Declaration of Helsinki.

\section{Procedures}

Studies were performed in a quiet room with an ambient temperature of $24^{\circ} \mathrm{C}$ and stable humidity. Each control subject and patient initially rested in a supine position for at least $45 \mathrm{~min}$ to ensure cardiovascular stability. Then we recorded a continuous electrocardiogram and non-invasive arterial blood pressure by radial artery tonometry (Colin Pilot ${ }^{\mathrm{TM}}$; Colin Medical Instruments, San Antonio, TX, USA). The blood pressure values were calibrated by reference to automatic oscillometric cuff measurement of the brachial artery. Mean cerebral blood flow velocity (CBFV) of the left proximal middle cerebral artery was assessed by transcranial Doppler sonography (Multidop XL; DWL, Sipplingen, Germany). The middle cerebral artery was insonated through the temporal window approximately $1 \mathrm{~cm}$ above the zygomatic arch at a depth of $35-55 \mathrm{~mm}$ using a $2-\mathrm{MHz}$ Doppler probe. After optimising the Doppler signal, the probe was attached to the skull at a fixed angle using a headband with an adjustable positioning system. Respiratory frequency was monitored by means of inductance plethysmography using two calibrated belts attached around the thorax and abdomen (Respitrace Calibrator; Ambulatory Monitoring, Ardsley, NY, USA) [13].

\section{Baroreceptor stimulation}

Responses of blood pressure and CBFV to baroreceptor stimulation were determined using a method previously described by Bernardi et al. [14]. The neck chamber consisted of a malleable lead collar edged with neoprene foam and was fitted to the anterior neck, over the carotid baroreceptors. Subatmospheric pressure was applied to the collar by means of a vacuum cleaner whose power output was regulated through a control unit. Pressure within the chamber was monitored with a pressure transducer (HugoSachs Elektronik, March, Germany) and could be set to oscillate between 0 and $30 \mathrm{mmHg}$ at $0.1 \mathrm{~Hz}$. Breathing was paced at 15 breaths $/ \min (0.25 \mathrm{~Hz})$ to avoid the effects of respiratory interference on cardiovascular autonomic modulation during the $0.1 \mathrm{~Hz}$ neck suction stimulation $[7,14,15]$. To familiarise the study participants with the respiratory frequency prior to the study, they were taught to follow visual and verbal signals to inspire and expire within $4 \mathrm{~s}$, i.e. close to their normal respiratory frequency.

An initial 5 min baseline recording was made during $0.25 \mathrm{~Hz}$ paced breathing without neck suction. The $0.1 \mathrm{~Hz}$ neck suction stimulation was then performed for $3 \mathrm{~min}$, with breathing paced at $0.25 \mathrm{~Hz}$ throughout $[7,14,15]$.

All data were digitised by a custom-made analogue-todigital converter at a sampling rate of $300 \mathrm{~Hz}$ and fed to a Macintosh PowerBook computer (Apple), manually cleaned from artefacts by linear interpolation and stored for off-line analysis [14]. A C-language program identified all QRS complexes in each sequence, located the peak of each $\mathrm{R}$ wave and calculated consecutive RR intervals. From the continuous waveforms of all parameters, beat-to-beat mean values were automatically calculated and interpolated linearly between adjacent values to construct a corresponding continuous time series [14].

From a $60 \mathrm{~s}$ interval at baseline and during $0.1 \mathrm{~Hz}$ neck suction, we calculated the mean and standard deviation of all biosignals.

Fluctuations in RR intervals, blood pressure, CBFV, respiration and pressure within the neck chamber were characterised by applying power spectrum analysis to these signals using an autoregressive algorithm with a linear detrending option and model order estimation according to Akaike information criteria $[14,16]$. Spectral power refers to the amount of variability of a signal (e.g. blood pressure or $\mathrm{CBFV}$ ) at a specific frequency. It is assessed as the magnitude of the oscillations at a given frequency and is expressed as units ${ }^{2}[17]$.

Autoregressive spectral analysis not only allows the quantitative evaluation of cardiovascular regulation in humans by assessing spontaneous oscillations in various biosignals, but also provides information about the transmission of neck suction-induced $0.1 \mathrm{~Hz}$ oscillations in various biosignals.

Neck suction applied at $0.1 \mathrm{~Hz}$ transmits oscillations at $0.1 \mathrm{~Hz}$ to the level of the RR interval, blood pressure and CBFV [14]. The response to $0.1 \mathrm{~Hz}$ neck suction at the level of blood pressure and CBFV is an index of sympathetic baroreceptor activation, and the response to $0.1 \mathrm{~Hz}$ neck suction at the RR interval level reflects sympathetic and/or vagal effects of baroreceptor stimulation on the heart [14]. We assessed the response to $0.1 \mathrm{~Hz}$ neck suction as the change in spectral power at $0.1 \mathrm{~Hz}$ in the RR 
interval, blood pressure and CBFV compared with the baseline value [14].

Cerebral regulation dampens the transmission of lowfrequency fluctuations of blood pressure onto the $\mathrm{CBFV}$ but not high-frequency blood pressure fluctuations [14]. To assess dynamic cerebral regulation, we calculated the transfer function gain and the phase shift between blood pressure and CBFV oscillations at $0.1 \mathrm{~Hz}$ as a measure of the transmission of the $0.1 \mathrm{~Hz}$ neck suction-induced blood pressure fluctuations on $\mathrm{CBFV}$, provided there was significant coherence between the two signals [11, 18, 19].

The coherence between blood pressure and CBFV signal oscillations might span from 0 (no association) to 1 (maximal association) [14]. Two signals were considered to have a stable phase relation for a given frequency of oscillation if coherence was above 0.5 . The gain was calculated between CBFV and blood pressure oscillations as the square root of the ratio of the $0.1 \mathrm{~Hz}$ power of CBFV divided by the $0.1 \mathrm{~Hz}$ power of blood pressure [11].

Statistical analysis

All values are presented as mean \pm SEM. Significance was assumed for $p$ values below 0.05. In each group, we compared differences between parameters at baseline and during $0.1 \mathrm{~Hz}$ neck suction by means of the $t$-test for dependent samples. In addition, we compared the parameters obtained in the patients with the parameters obtained in the controls using the $t$-test for independent samples. A commercially available statistical program (SPSS; SPSS, Chicago, IL, USA) was used for data analysis.

\section{Results}

Table 1 shows the resting cardiovascular state in the controls and patients. There were no significant differences in mean blood pressure, mean CBFV or their spectral powers between the controls and the patients with type 2 diabetes. Resting heart rate was, however, significantly higher in the patients with type 2 diabetes than in the healthy controls $(p=0.033)$. The transfer function gain between mean blood pressure and cerebral blood flow velocity did not differ significantly between patients and controls ( $p=0.353)$, but there was a significant difference in the phase relation between these two signals $(p=0.008)$.

Table 2 shows the responses to $0.1 \mathrm{~Hz}$ neck suction in the control subjects and patients. Application of $0.1 \mathrm{~Hz}$ neck suction did not significantly change the mean values of heart rate, blood pressure or CBFV in either the patients with type 2 diabetes or the control subjects. In the healthy volunteers, neck suction increased the $0.1 \mathrm{~Hz}$ powers of RR intervals, mean blood pressure and CBFV (Fig. 1). The measures of cerebral regulation (the transfer function gain between $0.1 \mathrm{~Hz}$ blood pressure and CBFV oscillations as well as the phase shift) remained stable during $0.1 \mathrm{~Hz}$ neck suction (Fig. 2). In the patients with type 2 diabetes, $0.1 \mathrm{~Hz}$ neck suction did not significantly increase the $0.1 \mathrm{~Hz}$ power of RR intervals, mean blood pressure and CBFV (Fig. 1). As in the control subjects, there were no changes in transfer function gain between $0.1 \mathrm{~Hz}$ blood pressure and CBFV oscillations during neck suction. In contrast, the phase shift between $0.1 \mathrm{~Hz}$ blood pressure and CBFV oscillations in the patients with type 2 diabetes was significantly lower during neck suction than at baseline (Fig. 2). Whereas the phase shift was lower during neck suction than at baseline in the patients, the absolute value during neck suction was not different from the value of the controls (Fig. 2).

\section{Discussion}

In the present study we extended our previous observations in 14 patients with type 2 diabetes in whom we had previously assessed cardiovagal and vasomotor responses to oscillatory neck suction [8]. To ensure that the patients had pronounced autonomic neuropathy, we included only the nine of the original 14 patients who definitely fulfilled the criteria of severe cardiac autonomic neuropathy, defined as pathological results in at least two of the standardised cardiovascular tests [12]. Cerebral blood flow velocity was recorded in the original study, but cerebral blood flow data were not analysed and included in our previous publication [8].
Table 1 Parameters at baseline in the 11 healthy controls and the nine patients with type 2 diabetes
All values are presented as
mean (SEM)
$B P$ Blood pressure, $C B F V$
cerebral blood flow velocity

\begin{tabular}{llll}
\hline Parameter & Controls & Patients & $t$-test \\
\hline RR interval $(\mathrm{ms})$ & $916.9(48.0)$ & $771.5(36.9)$ & $p=0.033$ \\
$0.1 \mathrm{~Hz}$ power of RR interval $\left(\mathrm{ms}^{2}\right)$ & $84.9(20.0)$ & $48.6(10.6)$ & $p=0.151$ \\
Mean BP (mmHg) & $93.1(2.8)$ & $91.0(2.6)$ & $p=0.595$ \\
Hz power of mean BP (mmHg) & $0.6(0.1)$ & $0.8(0.3)$ & $p=0.534$ \\
Mean CBFV (cm/s) & $39.8(3.6)$ & $32.7(4.2)$ & $p=0.217$ \\
$0.1 \mathrm{~Hz}$ power of mean CBFV $\left(\mathrm{cm}^{2} / \mathrm{s}^{2}\right)$ & $0.6(0.2)$ & $0.7(0.2)$ & $p=0.638$ \\
Transfer function gain $\left(\mathrm{cm} \mathrm{s}^{-1} \mathrm{mmHg}^{-1}\right)$ & $1.1(0.1)$ & $1.6(0.6)$ & $p=0.353$ \\
Phase shift (rad) & $-1.1(0.1)$ & $-1.7(0.2)$ & $p=0.008$ \\
\end{tabular}


Table 2 Responses to $0.1 \mathrm{~Hz}$ neck suction in the 11 healthy controls and the nine patients with type 2 diabetes

\begin{tabular}{|c|c|c|c|}
\hline Parameter & Controls & Patients & $t$-test \\
\hline $\mathrm{RR}$ interval (ms) & $6.55(9.00)$ & $1.61(7.36)$ & $p=0.685$ \\
\hline $\begin{array}{l}0.1 \mathrm{~Hz} \text { power of } \mathrm{RR} \\
\text { interval }\left(\mathrm{ms}^{2}\right)\end{array}$ & $110.23(53.27)$ & $93.62(65.79)$ & $p=0.845$ \\
\hline Mean BP (mmHg) & $-0.58(1.15)$ & $-0.22(0.92)$ & $p=0.812$ \\
\hline $\begin{array}{l}1.2 \mathrm{~Hz} \text { power of mean } \\
\mathrm{BP}\left(\mathrm{mmHg}^{2}\right)\end{array}$ & $0.83(0.25)$ & $0.02(0.25)$ & $p=0.040$ \\
\hline Mean CBFV $(\mathrm{cm} / \mathrm{s})$ & $-3.84(1.53)$ & $-1.72(0.88)$ & $p=0.275$ \\
\hline $\begin{array}{l}0.1 \mathrm{~Hz} \text { power of mean } \\
\mathrm{CBFV}\left(\mathrm{cm}^{2} / \mathrm{s}^{2}\right)\end{array}$ & $0.86(0.33)$ & $-0.08(0.15)$ & $p=0.025$ \\
\hline $\begin{array}{l}\text { Transfer function gain } \\
\left(\mathrm{cm} \mathrm{s}^{-1} \mathrm{mmHg}^{-1}\right)\end{array}$ & $0.09(0.15)$ & $-0.62(0.58)$ & $p=0.210$ \\
\hline Phase shift (rad) & $0.11(0.12)$ & $0.56(0.12)$ & $p=0.021$ \\
\hline
\end{tabular}

Data are presented as absolute differences between parameters at rest and during $0.1 \mathrm{~Hz}$ neck suction.

All values are presented as mean (SEM)

$C B F V$ Cerebral blood flow velocity

To our knowledge, this is the first study evaluating cerebrovascular regulation in type 2 diabetes by means of sinusoidal neck suction.

Cerebrovascular regulation refers to the intrinsic ability of the cerebral blood vessels to maintain a near-constant cerebral blood flow despite changes in perfusion pressure, provided the blood pressure remains within certain limits $(50-150 \mathrm{mmHg})$. Several mechanisms are thought to contribute to cerebrovascular regulation. These include a myogenic component as well as various neurohumoral, metabolic $\left(\mathrm{CO}_{2}\right.$ and $\left.\mathrm{H}^{+}\right)$and endothelial mechanisms [9]. The effect of sympathetic activation on cerebral circulation has been the object of extensive debate. Panerai et al. considered the sympathetic tone of cerebral arteries to be minimal under normal conditions [20]. However, Zhang et al. [21] and Ohta et al. [22] reported that the autonomic nervous system plays an essential role in the autoregulatory mechanisms of cerebral circulation. Diabetic patients have been shown to have endothelial dysfunction and other microvascular abnormalities. It is therefore possible that in addition to dysfunctional autonomic regulation of the blood vessels, diabetic patients have impaired mechanisms ensuring constancy of cerebral blood flow.

Our main finding was that, in patients with type 2 diabetes who have severe cardiac autonomic neuropathy, the mechanisms ensuring constancy of cerebral blood flow seem to be altered compared with healthy control subjects. Under resting conditions and during neck suction application, the mean value of CBFV, the power of CBFV oscillations and the transfer function gain did not differ between the controls and the patients.

The transfer function gain is well established for the assessment of cerebrovascular regulation and provides an

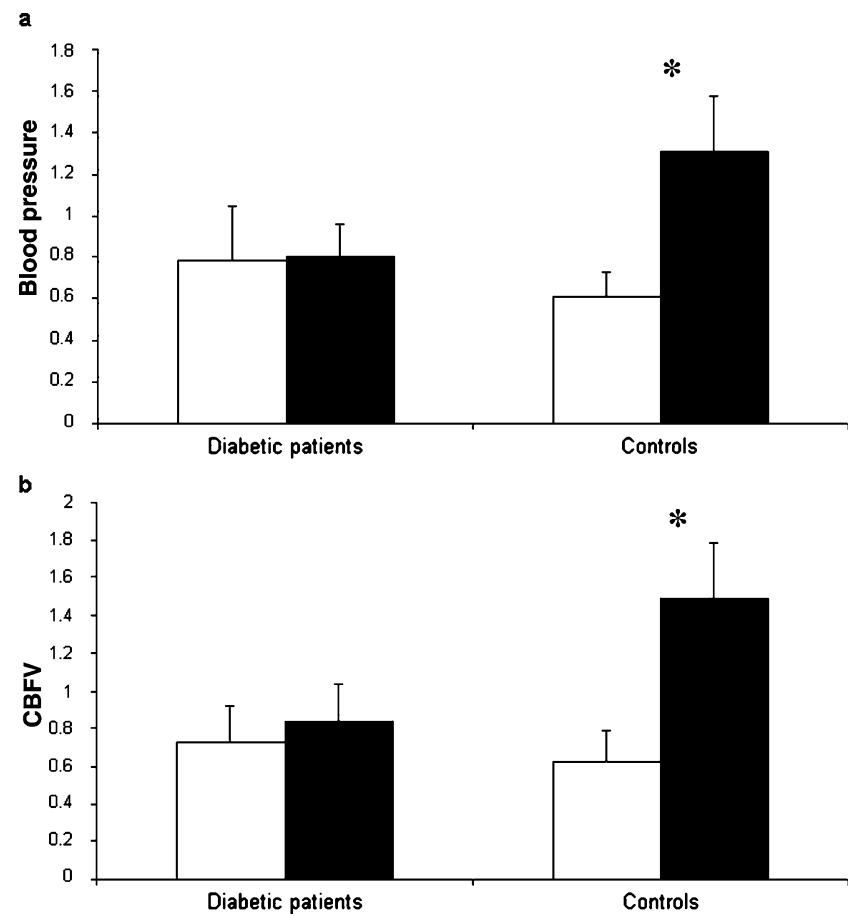

Fig. 1 Influence of $0.1 \mathrm{~Hz}$ neck suction on the sympathetically mediated $0.1 \mathrm{~Hz}$ powers of (a) mean blood pressure $(\mathrm{mmHg})$ and (b) mean cerebral blood flow velocity (CBFV) $\left(\mathrm{cm}^{2} \mathrm{~s}^{-2}\right)$ in the nine patients with type 2 diabetes and the 11 healthy controls. In the healthy controls, neck suction typically increased the $0.1 \mathrm{~Hz}$ powers of blood pressure and CBFV. In the patients, however, $0.1 \mathrm{~Hz}$ neck suction did not induce the typical increase in the $0.1 \mathrm{~Hz}$ powers of blood pressure and $\mathrm{CBFV}$, suggesting deficient sympathetic vasomotor control. Open bars Blood pressure and CBFV before $0.1 \mathrm{~Hz}$ neck suction; filled bars blood pressure and CBFV during $0.1 \mathrm{~Hz}$ neck suction. ${ }^{*} p<0.05$

analysis of cerebrovascular responses to sinusoidal neck suction. The gain was determined from the transfer function of mean blood pressure oscillations as the input signal and mean CBFV oscillations as the output signal. A low transfer function gain indicates strong buffering of blood pressure fluctuations by cerebral resistance vessels, i.e. intact cerebrovascular regulation $[11,19]$. The transfer function gain reflects the extent to which there is a transmission of blood pressure fluctuations onto CBFV fluctuations or a buffering of such fluctuations by the mechanisms ensuring constancy of cerebral blood flow [11, 19]. As the patients showed values of the transfer function gain similar to those of the healthy controls, the ability of the cerebral blood vessels to buffer fluctuations in systemic blood pressure seems to be intact in the patients with type 2 diabetes. However, baroreceptor stimulation did not increase the magnitude of blood pressure oscillations in the patients with type 2 diabetes. Therefore, a subsequent change in the power of CBFV would not be expected. Thus, the transfer function gain is probably not an adequate method of assessing cerebrovascular regulation in patients with type 2 diabetes. 


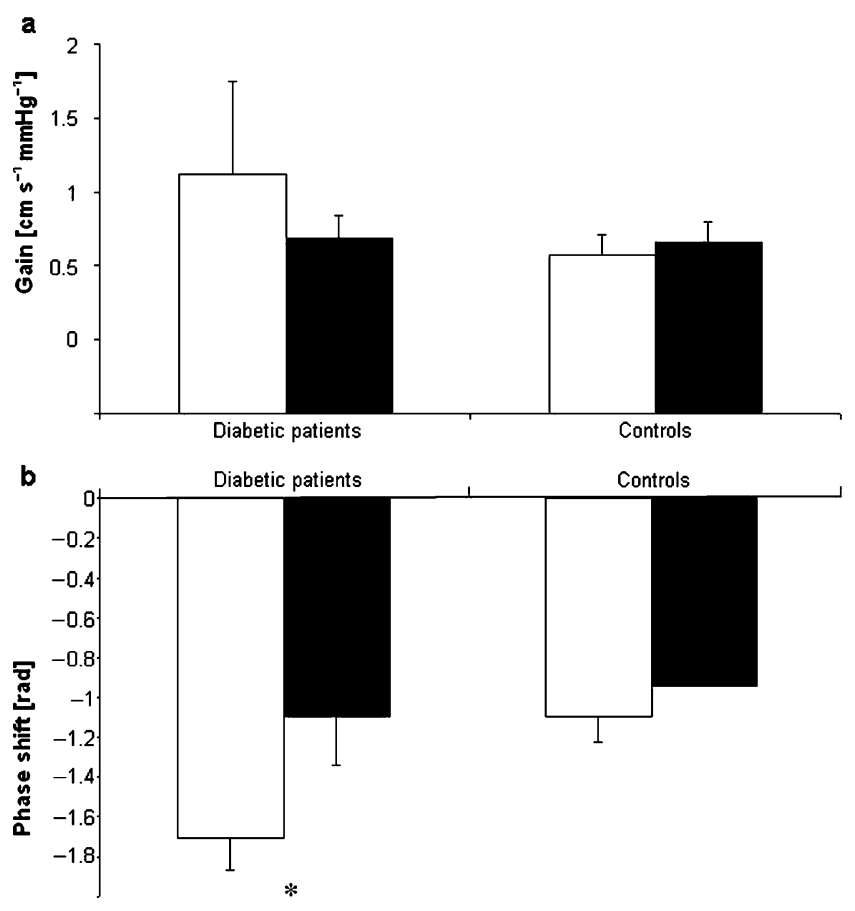

Fig. 2 Transfer function gain (a) and phase shift (b) between mean blood pressure and mean cerebral blood flow velocity oscillations at $0.1 \mathrm{~Hz}$ in 11 healthy controls and nine patients with type 2 diabetes before and during $0.1 \mathrm{~Hz}$ neck suction. In the patients, $0.1 \mathrm{~Hz}$ neck suction did not change the transfer function gain since sympathetic dysfunction prevented any increase in the spectral power of blood pressure at $0.1 \mathrm{~Hz}$, i.e. in the input signal of cerebral regulation. The phase shift analysis, however, revealed a decrease in the patients, indicating altered cerebral regulation. Open bars Gain and phase shift before $0.1 \mathrm{~Hz}$ neck suction; filled bars gain and phase shift during $0.1 \mathrm{~Hz}$ neck suction. ${ }^{*} p<0.05$

In addition, the phase relation between oscillations in mean blood pressure and mean $\mathrm{CBFV}$ can be used to determine the quality of the mechanisms ensuring constancy of cerebral blood flow. Diehl et al. showed that blood pressure oscillations induced by metronomic breathing at 6 cycles/min generate oscillations in the CBFV signal at the same frequency, which are not only dampened but also shifted to the left because of the high-pass filter characteristics of the mechanisms ensuring constancy of cerebral blood flow [11, 18]. A rise in blood pressure - induced by metronomic breathing - normally induces counter-regulation of the vascular resistance bed with vasoconstriction. Because of the vasoconstriction, $\mathrm{CBFV}$ has reached its maximum before blood pressure is at its highest level. While blood pressure approaches its maximum, there is already a slowing of CBFV. When blood pressure starts to decline, CBFV declines even faster as the response of the cerebral resistance vessels to the preceding rise in blood pressure persists. When blood pressure declines further, the cerebrovascular system counteracts any further decrease in $\mathrm{CBFV}$ by vasodilatation. Again, the minimum CBFV precedes the minimum blood pressure. Consequently, there is a recovery of CBFV before the next increase in blood pressure. The relation between the leading $\mathrm{CBFV}$ and lagging blood pressure oscillations can be described by calculating the phase shift between the two signals $[11,18]$. Analysis of this phase shift, i.e. the time delay between the oscillations in blood pressure and $\mathrm{CBFV}$, revealed some differences in the behaviour of the mechanisms ensuring constancy of cerebral blood flow between the controls and the patients with type 2 diabetes. At rest, the phase shift between mean blood pressure and CBFV was greater in the patients than in the controls. Baroreflex stimulation with $0.1 \mathrm{~Hz}$ neck suction decreased the phase shift significantly in the patients, but only slightly in the controls. According to Diehl et al., the phase shift between blood pressure and CBFV oscillations during deep breathing at 6 breaths $/ \mathrm{min}$ in healthy subjects is between 30 and $90^{\circ}$ (equal to 0.52 and $1.57 \mathrm{rad}$ ). A decrease of the phase angle below such normal values indicates a more passive behaviour of the cerebral vessel bed and might indicate impaired cerebrovascular regulation [18]. In patients with high-grade cerebral artery stenoses, the phase angle between the two biosignals is reduced or even absent, i.e. the CBFV oscillations are driven passively by blood pressure oscillations and there is no longer any filtering by the mechanisms ensuring constancy of cerebral blood flow [18].

The phase shift results in our study are at slight variance with the results reported by Cencetti et al. [23]. During head-up tilt, Cencetti et al. observed a significant reduction in phase shift in their controls [23]. This shortening of phase shift was ascribed to sympathetic activation and subsequent sympathetically mediated vessel stiffening [23]. In our controls, sympathetic challenge with $0.1 \mathrm{~Hz}$ neck suction also decreased the phase shift, but the decrease was only slight and not significant. Cerebrovascular regulation prevented substantial changes in the phase shift. Another possible explanation for this slight difference might be the different stimuli used in the two studies. In our study, we used sinusoidal neck suction, which is a repetitive stimulus, while Cencetti et al. evaluated cerebrovascular responses to continuous orthostatic challenge.

Although the phase shift in the controls differs only slightly between the two studies, major differences are evident in the results of the patients. Cencetti et al. reported a lower phase shift at rest in the patients than the controls [23]. In contrast, our patients had a bigger phase shift before neck suction than the controls. We assume that our patients had diabetic autonomic neuropathy at a later stage of the disease and the stiffening, i.e. sympathetic tone, was already more compromised than in the patients of Cencetti et al. Because of the poor sympathetic outflow, the phase shift is already rather high at rest. With $0.1 \mathrm{~Hz}$ neck suction the cerebral regulatory system of our diabetic patients 
cannot maintain the initial phase shift. Perhaps, there is a relative, positive forward shift as a result of passive transmission of blood pressure fluctuations, as described by Cencetti et al. This relative passive component may decrease the negative phase shift values seen at rest.

Our finding of a greater phase shift in patients with type 2 diabetes under resting conditions, along with the decrease in the phase shift during $0.1 \mathrm{~Hz}$ neck suction, might indicate altered cerebrovascular regulation during baroreceptor stimulation in patients with type 2 diabetes. Our diabetic patients were previously shown to have impaired cardiovagal and sympathetic vasomotor responses to baroreceptor stimulation [8]. As a novel finding extending beyond our previous ones, we showed that phase shift analysis during $0.1 \mathrm{~Hz}$ neck suction is suited to identifying subtle impairment of cerebrovascular regulation in patients who have type 2 diabetes and autonomic neuropathy.

\section{References}

1. Kothari V, Stevens RJ, Adler AI et al (2002) UKPDS 60: risk of stroke in type 2 diabetes estimated by the UK Prospective Diabetes Study risk engine. Stroke 33:1776-1781

2. Grau AJ, Weimar C, Buggle F et al (2001) Risk factors, outcome, and treatment in subtypes of ischemic stroke: the German stroke data bank. Stroke 32:2559-2566

3. Toyry JP, Niskanen LK, Lansimies EA, Partanen KP, Uusitupa MI (1996) Autonomic neuropathy predicts the development of stroke in patients with non-insulin-dependent diabetes mellitus. Stroke 27:1316-1318

4. Mankovsky BN, Piolot R, Mankovsky OL, Ziegler D (2003) Impairment of cerebral autoregulation in diabetic patients with cardiovascular autonomic neuropathy and orthostatic hypotension. Diabet Med 20:119-126

5. Ewing DJ, Campbell IW, Clarke BF (1980) The natural history of diabetic autonomic neuropathy. Q J Med 49:95-108

6. Dutsch M, Hilz MJ, Neundorfer B (2001) Diabetic autonomic neuropathy. Fortschr Neurol Psychiatr 69:423-438

7. Brown CM, Dutsch M, Michelson G, Neundorfer B, Hilz MJ (2002) Impaired cardiovascular responses to baroreflex stimulation in open-angle and normal-pressure glaucoma. Clin Sci (Lond) 102:623-630

8. Sanya EO, Brown CM, Dutsch M, Zikeli U, Neundorfer B, Hilz MJ (2003) Impaired cardiovagal and vasomotor responses to baroreceptor stimulation in type II diabetes mellitus. Eur J Clin Invest 33:582-588

9. Hilz MJ, Stemper B, Heckmann JG, Neundörfer B (2000) Mechanisms of cerebral autoregulation, assessment and interpretation by means of transcranial doppler sonography. Fortschr Neurol Neurochir Psych 68:398-412

10. Lagi A, Laffi G, Cencetti S et al (2002) Impaired sympathetic regulation of cerebral blood flow in patients with cirrhosis of the liver. Clin Sci (Lond) 103:43-51

11. Hilz MJ (2002) Quantitative autonomic functional testing in clinical trials. In: Brown R, Bolton C, Aminoff M (eds) Neuromuscular function and disease. W.B. Saunders, Philadelphia, pp 1899-1929

12. Ewing DJ, Clarke BF (1982) Diagnosis and management of diabetic autonomic neuropathy. Br Med J 285:916-918

13. Marthol H, Werner D, Brown CM, Hecht M, Daniel WG, Hilz MJ (2005) Enhanced external counterpulsation does not compromise cerebral autoregulation. Acta Neurol Scand 111:34-41

14. Bernardi L, Bianchini B, Spadacini G et al (1995) Demonstrable cardiac reinnervation after human heart transplantation by carotid baroreflex modulation of RR interval. Circulation 92:2895-2903

15. Brown CM, Hecht MJ, Weih A, Neundorfer B, Hilz MJ (2003) Effects of age on the cardiac and vascular limbs of the arterial baroreflex. Eur J Clin Invest 33:10-16

16. Burr RL, Cowan MJ (1992) Autoregressive spectral models of heart rate variability. Practical issues. J Electrocardiol 25:224-233

17. Bernardi L, Salvucci F, Suardi R et al (1990) Evidence for an intrinsic mechanism regulating heart rate variability in the transplanted and the intact heart during submaximal dynamic exercise? Cardiovasc Res 24:969-981

18. Diehl RR, Linden D, Lucke D, Berlit P (1995) Phase relationship between cerebral blood flow velocity and blood pressure. A clinical test of autoregulation. Stroke 26:1801-1804

19. Zhang R, Zuckerman JH, Giller CA, Levine BD (1998) Transfer function analysis of dynamic cerebral autoregulation in humans. Am J Physiol 274:H233-H241

20. Panerai RB, Dawson SL, Eames PJ, Potter JF (2001) Cerebral blood flow velocity response to induced and spontaneous sudden changes in arterial blood pressure. Am J Physiol Heart Circ Physiol 280:H2162-H2174

21. Zhang R, Zuckerman JH, Iwasaki K, Wilson TE, Crandall CG, Levine BD (2002) Autonomic neural control of dynamic cerebral autoregulation in humans. Circulation 106:1814-1820

22. Ohta K, Gotoh F, Shimazu K et al (1991) Locus coeruleus stimulation exerts different influences on the dynamic changes of cerebral pial and intraparenchymal vessels. Neurol Res 13:164-167

23. Cencetti S, Lagi A, Cipriani M, Fattorini L, Bandinelli G, Bernardi L (1999) Autonomic control of the cerebral circulation during normal and impaired peripheral circulatory control. Heart $82: 365-372$ 